\title{
Neural Network based Road Sign Recognition
}

\author{
Sanjit Kumar Saha \\ Lecturer \\ Department of Computer Science \\ and Engineering \\ Comilla University, Comilla, \\ Bangladesh
}

\author{
Dulal Chakraborty \\ Lecturer \\ Department of Information and \\ Communication Technology \\ Comilla University, Comilla, \\ Bangladesh
}

\author{
Md. Al-Amin Bhuiyan \\ Professor \\ Department of Computer Science \\ and Engineering \\ Jahangirnagar University, Savar, \\ Dhaka, Bangladesh
}

\begin{abstract}
A recent surge of interest is to recognize Road Signs. Signs are visual languages that represent some special circumstantial information of environment. They provide important information for guiding, warning people to make their movements safer, easier and more convenient. This thesis presents a hybrid neural network solution for Road sign recognition which combines local image sampling and artificial neural network. The method is based on BAM for dimensional reduction and multi-layer perception with backpropagation algorithm has been used for training the network. It has been found from practical observations that the number of iterations required to train the network is enormous. The capability of recognition of a neural network increases with increasing the training accuracy. For this process each sign is converted to a designated $M \times N$ feature matrix. These feature matrices of signs are then fed into the neural network as input patterns. The neural network is trained with the set of input patterns of the digits to acquire separate knowledge corresponding to each Road sign. In order to justify the effectiveness of the system, different test patterns of the signs are used to verify the system. Experimental results demonstrate that the system is capable of recognizing Road signs with $98 \%$ accuracy.
\end{abstract}

\section{Keywords}

Road Sign Recognition, Hybrid Network, Neural Network.

\section{INTRODUCTION}

Driving is a task based almost entirely on visual information processing. The road signs and traffic signals define a visual language interpreted by drivers. Road signs carry many information necessary for successful driving - they describe the current traffic situation, define right-of-way, prohibit or permit certain directions, warn about risky factors etc. Road signs also help drivers with navigation.

Road signs provide important information for drivers about road condition and hazards. Their discriminating shape and colors make them easily recognizable by humans. Same factors can help development of a vision based RSR system. Beside the application of RSR in autonomous vehicles, it can also serve as an assistant driver (e.g. when combined with speedometer output) to notify the driver about approaching a Road sign (e.g. even before driver sees it) or his risky behavior (like driving above the speed limit).

This paper proposes a recognition algorithm of mandatory road signs shown below:

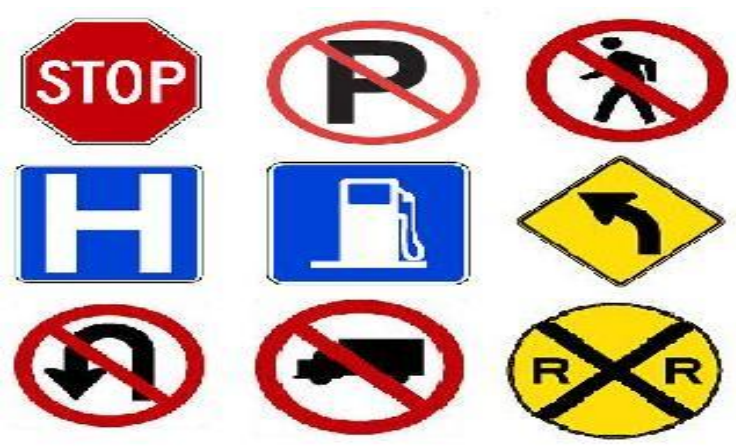

Figure 1. Mandatory Road Signs

For recognition, each Road sign image is represented as $64 \times 64$ pixels, as shown in Figure 2.

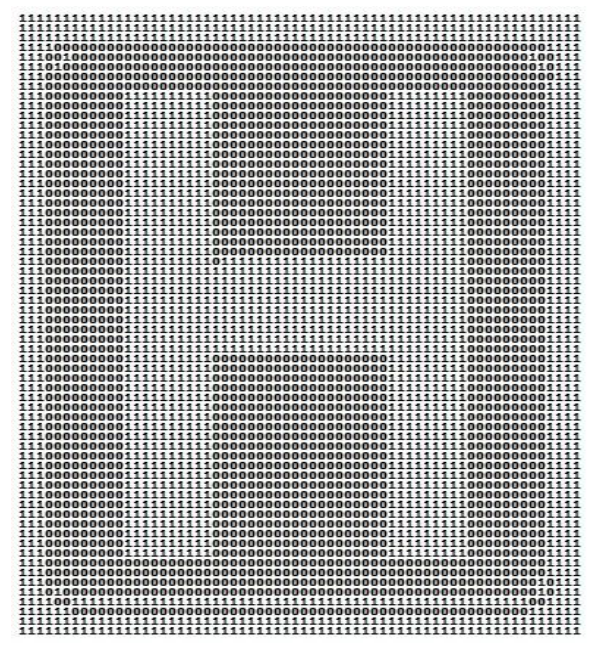

Figure 2. Formation of 'HOSPITAL'

\section{PREVIOUS WORKS AND MOTIVATION}

Mueller et al. [2] developed a computer vision system for the detection of objects in outdoor scenes. Piccioli et al. [3] developed a road sign detection system by analyzing color as a priori information to limit the possible locations of signs in the image applying a cross-correlation technique. Novovicova et al. [4] used a Laplace Kernel classifier in the decision tree for road sign classification, despite some difficulties in the recognition of square signs in urban areas, where lots of horizontal and vertical lines exist in the image. Yuille et al. [5] developed an approach to detect only stop signs by correcting the color of the ambient illumination, locating the boundaries of the signs and mapping the sign into a front 
parallel position before reading the sign. De La Escalera et al. [6] started with color matching, in which they looked for patterns in specific relationships that correspond to triangular, rectangular or circular signs. Lauziere et al. [7] used a physics-based approach for sign recognition, but this approach required keeping in memory the changes in the model parameter to accommodate the natural variation of illumination. Template matching methods that were used face some problems in case of noise in analog or gray scale images, improper illumination in the images, sampling and quantization noise and presence of the shadows in the images. After surveying different research works, the objective of our thesis is to represent a fast and robust system for road sign recognition which is a combination of a local image sample representation and a hybrid network.

\section{PREPROCESSING}

Road signs are being preprocessed by following a sequence of operations: Capturing, Binary image conversion.

\subsection{Capturing}

Acquisition of a Road sign is done by using a camera (Sony Cyber shot). In this investigation, some mandatory road signs had been employed for image database in BMP type file. During capturing operation, standardization and geometrical normalization (size and direction) of the images had been performed.

During analysis images were being made as $64 \times 64$ pixels resolution, as shown in Figure 3.

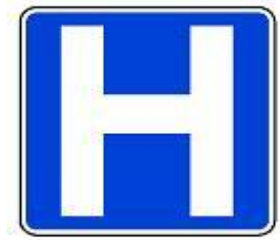

Figure 3. Capturing a Road sign image

\subsection{Binary Image Conversion}

The original image was obviously a color image. It was first converted into grayscale image and then binarized because it is easy to match patterns in binary format for sign recognition. Each sign is a Matrix representation of some values (ranges 0 to 255). These can be represented in binary form (0 and 1). The conversion process is shown in Figure 4:
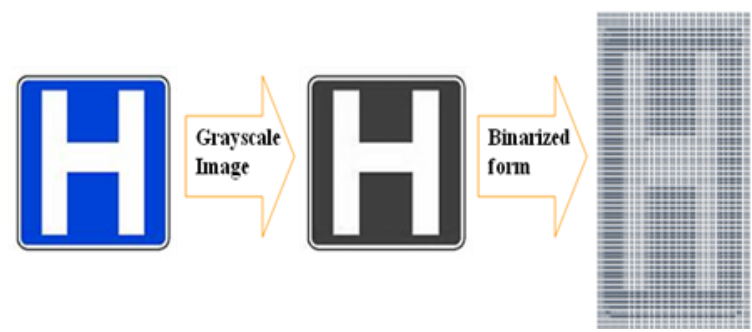

Figure 4. Image Conversion

Experiments were carried out on a Pentium IV $2.4 \mathrm{GHz}$ PC with 256 MB RAM. The algorithm had been implemented using $\mathrm{C}$.

\section{HYBRID NEURAL NETWORK}

A hybrid neural network is composed of two neural networks. One is BAM NN and the other is bidirectional NN. Bidirectional Associative Memory (BAM) has used for dimensional reduction of the feature matrix to make the recognition faster and more efficient. The network architecture for the hybrid neural network is shown in Figure 5 .

After preprocessing, the Road signs are engraved in a $64 \times 64$ grid and fed into the input layer of the neural network as the input feature vectors or training patterns. The grid patterns are, therefore, represented as vectors of 4096 components (if the pixel in the grid is shaded, the vector component is 1 otherwise it is 0 ). The number of neurons in the hidden layers are 50 (50\% of the input layer) for each. Since a total of 64 signs including both mandatory and others are being classified, so the number of neurons in the output layer is 64 . With 64 neurons in the output layer, we can represent a 6 bit code $\left(2^{6}=64\right)$ to classify each target output. So the target outputs are 000000 to 111111 , corresponding to each sign.

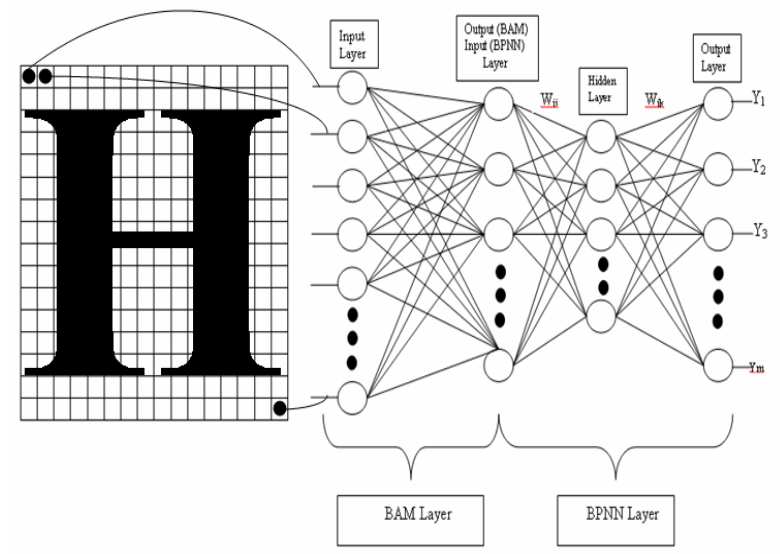

Figure 5. Hybrid Neural Network Topology

Processing Hybrid NN

After training, we have to do some simple calculation in order to reorganize Road signs. In the process of reorganization we need not train all the road signs.

\section{EXPERIMENTAL RESULTS \& PERFORMANCE}

The road sign recognition phase is most important and complicated phase. Hence, the sign is $64 \times 64$ pixels image. Each sign has a feature matrix of 4096 elements in it. Each element is nothing but binary values ( 0 and 1$)$.

Hence, in our experiment the number of neurons in input layer was 4096, neurons in input layer for BPNN and output layer for BAM was 16 , number of neurons in hidden layer was 10 , and finally the neurons in output layer was 6 . The number of neurons in hidden layer can vary from $50 \%$ to $70 \%$ of its input neurons.

In order to justify the performance of the neural network, various experiments were carried out. All experiments were performed with 8 training images and a test images for each sign. There was no overlap between the training and test image sets. The back-propagation neural network was trained using the default learning parameter settings (learning rate 0.3 , threshold 1) for 75 epochs. Finally, the neural network was used to recognize signs separately. 


\subsection{Recognition of Normal Images}

Figure 6 shows the snapshot of the program output of a normal image, without noise and light variation.

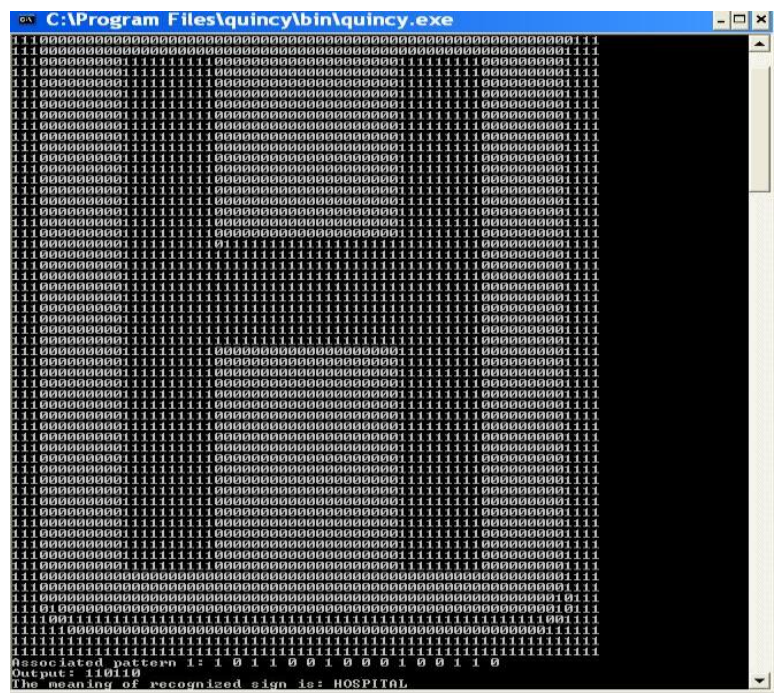

Figure 6. Recognition of sign ('HOSPITAL')

The hybrid network helps to recognize sign. After the recognition of these the meaning of the captured sign was 'HOSPITAL'.

Similarly,

\begin{tabular}{|c|c|c|c|c|}
\hline $\begin{array}{l}\text { Sample } \\
\text { Input }\end{array}$ & STOP & 2 & & 林 \\
\hline $\begin{array}{l}\text { Sample } \\
\text { Output }\end{array}$ & STOP & $\begin{array}{c}\text { NO } \\
\text { PARKIN } \\
\text { G }\end{array}$ & $\begin{array}{c}\text { NO } \\
\text { PEDESTR } \\
\text { AIN }\end{array}$ & $\begin{array}{c}\text { SCHOO } \\
\text { L }\end{array}$ \\
\hline $\begin{array}{l}\text { Sample } \\
\text { Input }\end{array}$ & & & & $B$ \\
\hline $\begin{array}{l}\text { Sample } \\
\text { Output }\end{array}$ & $\begin{array}{l}\text { NO U } \\
\text { TERN }\end{array}$ & $\begin{array}{c}\text { NO } \\
\text { BICYCLE }\end{array}$ & GAS & $\begin{array}{c}\text { NO } \\
\text { RIGHT } \\
\text { TURN }\end{array}$ \\
\hline
\end{tabular}

\subsection{Images with Different Lighting \\ Conditions}

Several experiments were done in different lighting conditions, such as (i) Dark lighting condition (ii) Sunny lighting condition (iii) Cloudy. For each of the stages, some sorts of preprocessing were performed using histogram equalization technique. For example,

\subsubsection{Dark lighting condition}

The experiment was performed by capturing an image in dark lighting condition and enhanced the captured image using histogram equalization as follows:
Preprocessing:
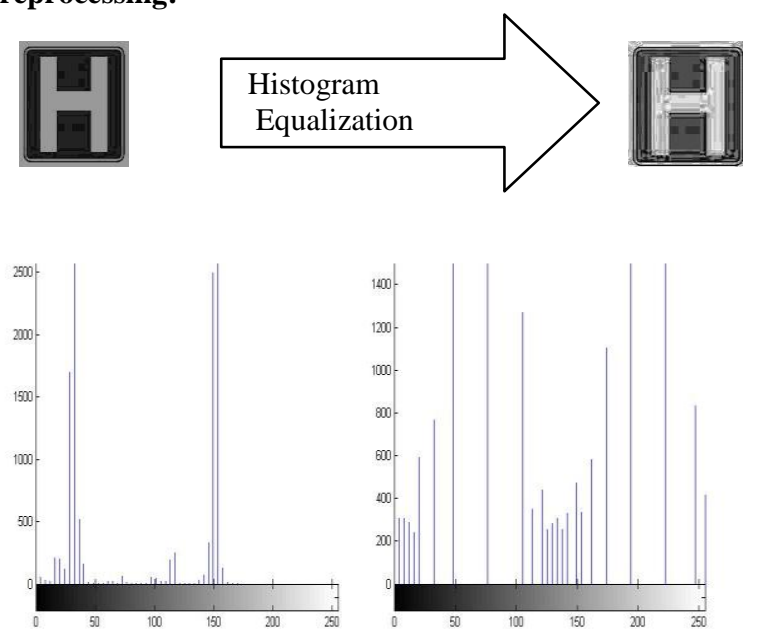

The processed image was then recognized using the hybrid network, as shown in Figure 7.

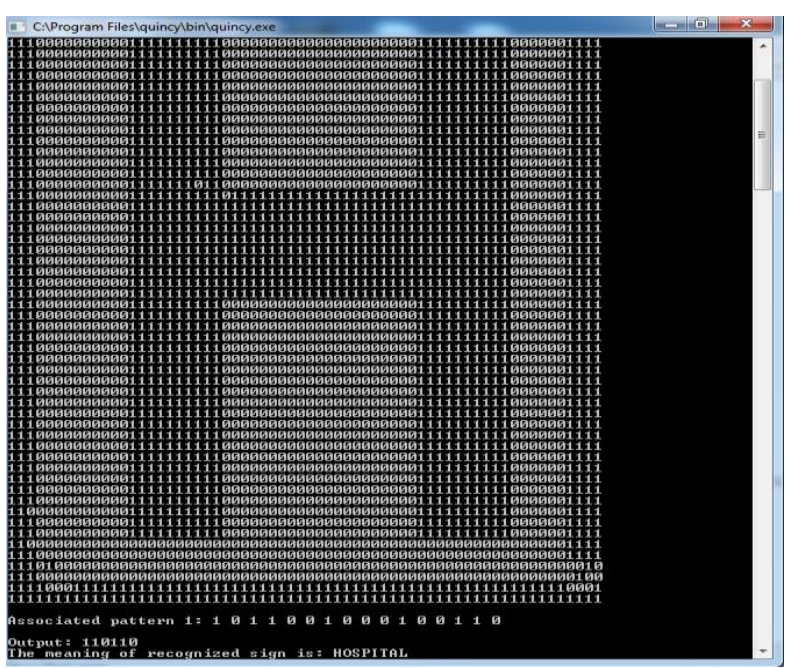

Figure 7. Recognition of sign in dark condition ('HOSPITAL')

It seems that the hybrid network was able recognized the captured road sign in dark lighting condition successfully and displayed that the meaning of the recognized sign is "HOSPITAL".

\subsubsection{Sunny lighting condition}

The experiment was performed by capturing an image in sunny lighting condition and enhanced the captured image using histogram equalization as follows: 
Preprocessing:

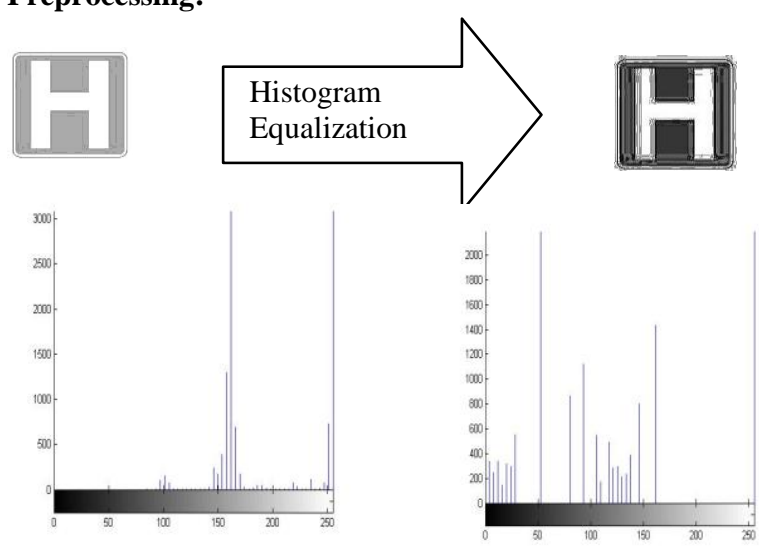

The processed image was then recognized using the hybrid network, as shown in Figure 8.

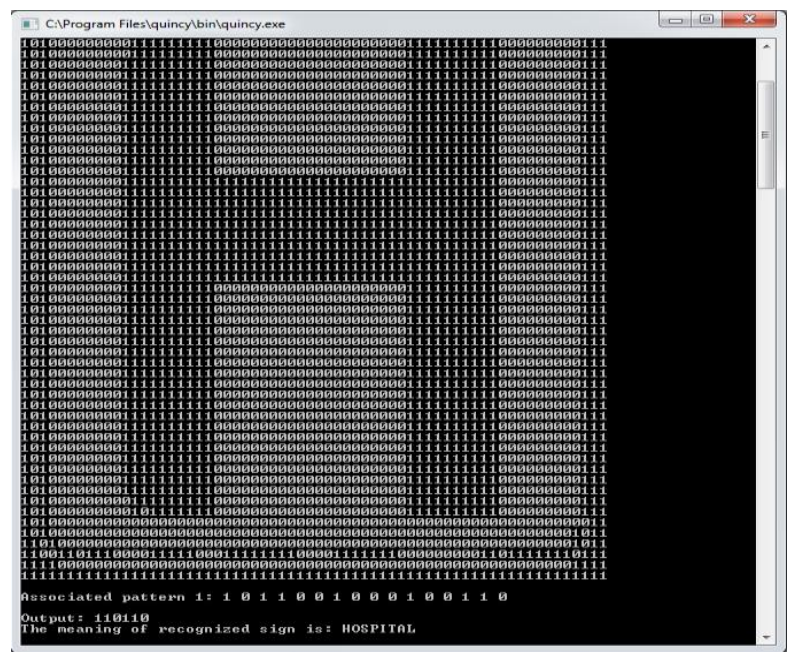

Figure 8. Recognition of sign in sunny condition ('HOSPITAL')

It seems that the hybrid network was recognized the captured road sign in sunny lighting condition successfully and displayed that the meaning of the recognized sign is "HOSPITAL".

\subsubsection{Cloudy lighting condition}

An image was captured in cloudy lighting condition and performed the preprocessing using histogram equalization as follows:

\section{Preprocessing:}

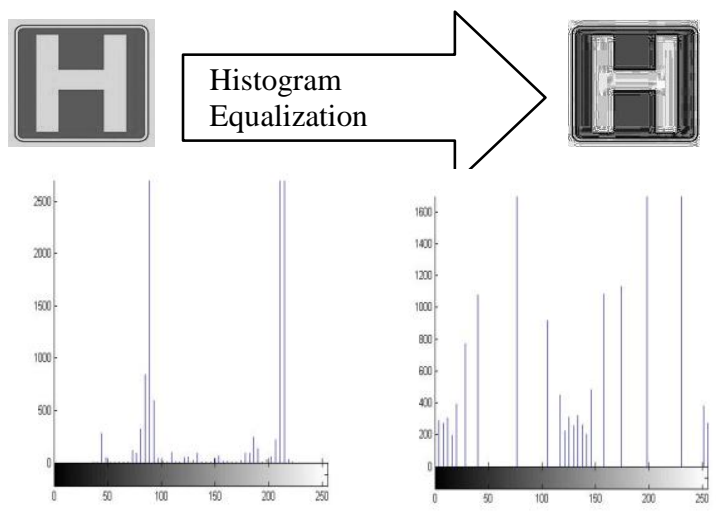

The processed image was then recognized using the hybrid network, as shown in Figure 9.

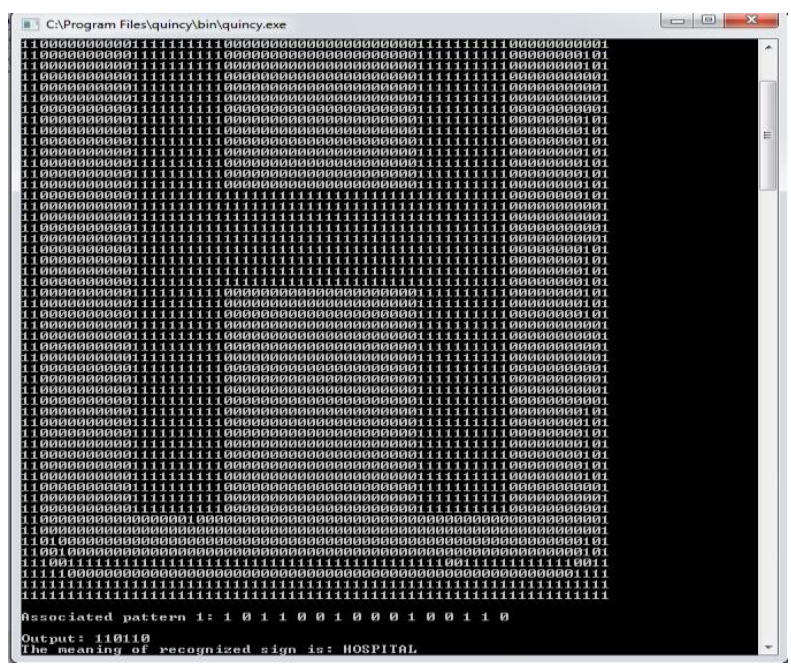

Figure 9. Recognition of sign in cloudy condition ('HOSPITAL')

It seems that the hybrid network was recognized the captured road sign in cloudy lighting condition successfully and displayed that the meaning of the recognized sign is "HOSPITAL".

The system had been checked using 60 signs in different lighting conditions (20 signs in dark lighting conditions, 20 signs in sunny lighting conditions and 20 signs in cloudy lighting conditions). Table 1 shows the comparison results based on the recognition accuracy from which our method is seen to be superior.

Table 1. Comparison results based on recognition accuracy

\begin{tabular}{|c|c|c|c|}
\hline $\begin{array}{c}\text { Network } \\
\text { Type }\end{array}$ & Dark & Sunny & Cloudy \\
\hline $\begin{array}{c}\text { BP with } \\
\text { BAM }\end{array}$ & $90 \%$ & $98 \%$ & $96 \%$ \\
\hline BP & $71 \%$ & $84 \%$ & $78 \%$ \\
\hline
\end{tabular}

\subsection{Recognition for Noisy Images}

In order to justify the robustness of the method, different kinds of noisy images were included in different experiments. Three different types of noises were includes like (i) Gaussian noise (ii) Poisson noise and (iii) Speckle noise. For example,

\subsubsection{Gaussian noise}

The PDF of a Gaussian random variable ${ }^{[14]}, \mathrm{z}$, is given by

$f(z)=\frac{1}{\sqrt{2 \pi} \sigma} e^{-\frac{(z-z)^{2}}{z \sigma^{2}}}$

Where, $\boldsymbol{z}$ represents intensity, $\overline{\mathbf{z}}$ is the mean value of $\boldsymbol{z}$ and $\sigma$ is its standard deviation. The standard deviation squared, $\sigma^{2}$, is called the variance of $\boldsymbol{Z}$.

The experiment was performed by imposing Gaussian noise in a sign with mean zero and variance 0.01 and observed that the hybrid network can recognize the noisy sign successfully. 


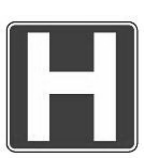

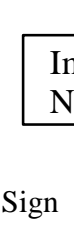

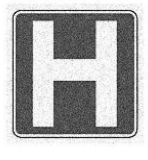

After Imposing Gaussian

Typical Road Sign Noise

The image was then recognized using the hybrid network, as shown in Figure 10.

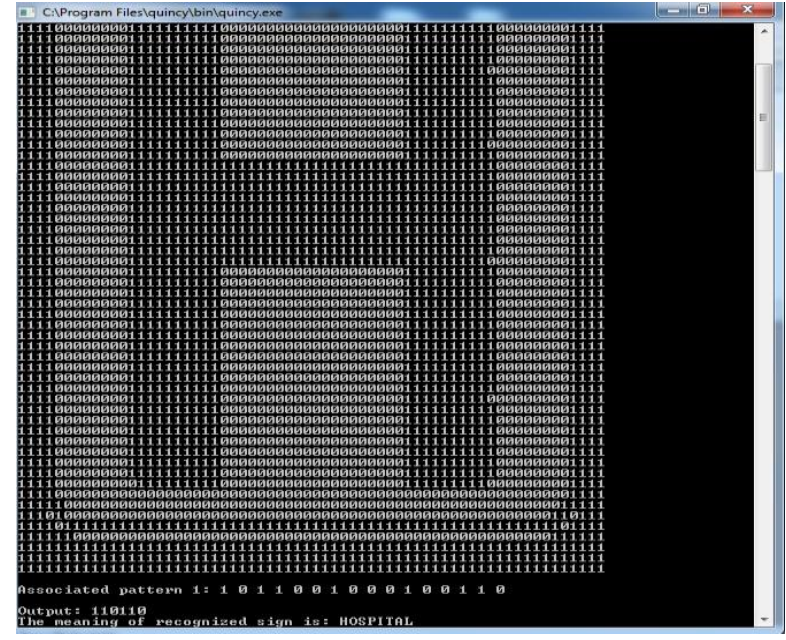

Figure 10. Recognition of Gaussian noisy sign ('HOSPITAL')

\subsubsection{Poison noise}

If the expected number of occurrences in a given interval is $\lambda$, then the probability that there are exactly $k$ occurrences $(k$ being a non-negative integer, $k=0,1,2 \ldots)$ is equal to

$f(k, \lambda)=\frac{\lambda^{k} e^{-\lambda}}{k !}$

Where, $e$ is the base of the natural logarithm $(e=2.71828 \ldots)$, $k$ is the number of occurrences of an event - the probability of which is given by the function, $k$ ! is the factorial of $k, \lambda$ is a positive real number, equal to the expected number of occurrences during the given interval. For instance, if the events occur on average 4 times per minute, and one is interested in the probability of an event occurring k times in a 10 minute interval, one would use a Poisson distribution as the model with $\lambda=10 \times 4=40$. As a function of $k$, this is the probability mass function.

The experiment was performed by imposing Poison noise in a sign observed that the hybrid network can recognize the noisy sign successfully.

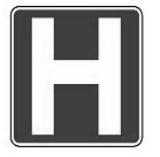
Imposing Poisson
Noise

Typical Road Sign

After Imposing Poisson Noise
The image was then recognized using the hybrid network, as shown in Figure 11.

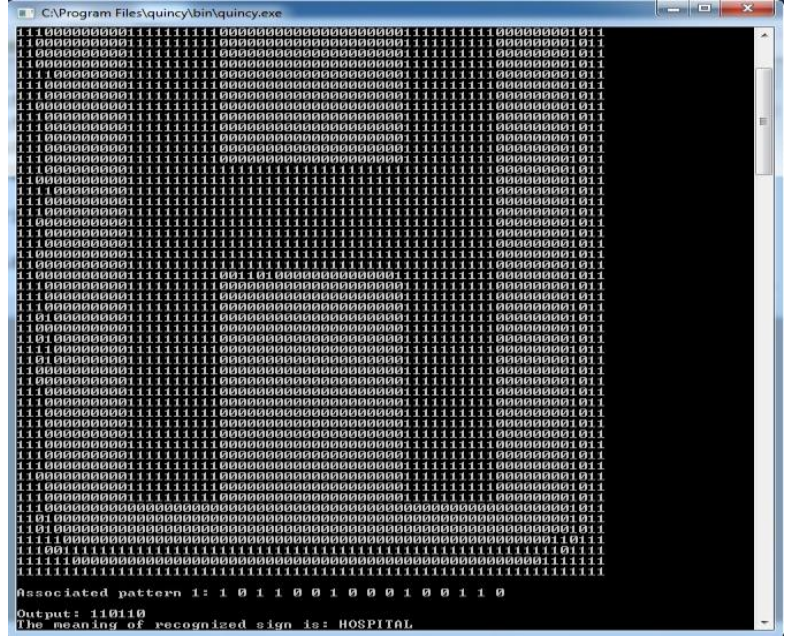

Figure 11. Recognition of Poisson noisy sign ('HOSPITAL')

\subsubsection{Speckle noise}

The experiment was performed by imposing Speckle noise in a sign with mean zero and variance 0.04 and observed that the hybrid network can recognize the noisy sign successfully.

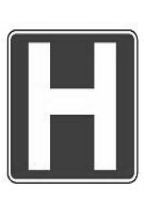

Typical Road Sign

Noise

The image was then recognized using the hybrid network, as shown in Figure 12.

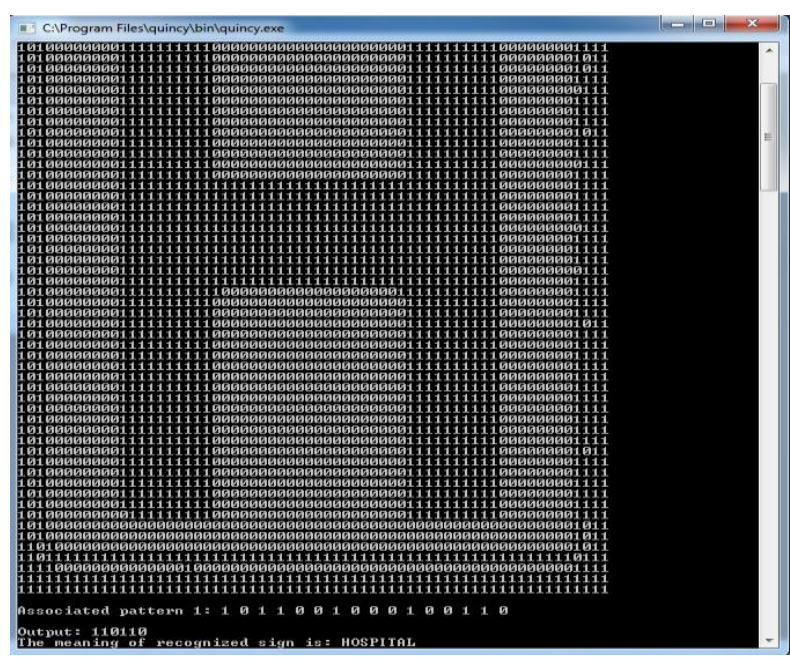

Figure 12. Recognition of Speckle noisy sign ('HOSPITAL')

The experiment was performed by changing the values of different parameters of each imposed noise. The system had been checked using 60 signs by imposing different noise (20 Gaussian noisy sign, 20 Poisson noisy sign and 20 Speckle noisy sign). Table 2 shows the comparison results based on the recognition accuracy from which our method is seen to be superior. 
Table 2. Comparison results based on recognition accuracy

\begin{tabular}{|c|c|c|c|}
\hline $\begin{array}{c}\text { Network } \\
\text { Type }\end{array}$ & Gaussian & Poisson & Speckle \\
\hline $\begin{array}{c}\text { BP with } \\
\text { BAM }\end{array}$ & $96 \%$ & $98 \%$ & $94 \%$ \\
\hline BP & $81 \%$ & $85 \%$ & $80 \%$ \\
\hline
\end{tabular}

In summary, the system had been checked using 180 signs (60 typical signs, 60 different lighting condition signs and 60 noisy signs). Table 3 shows the comparison results based on the recognition accuracy from which our method is seen to be superior.

Table 3. Comparison results based on recognition accuracy

\begin{tabular}{|c|c|c|c|}
\hline $\begin{array}{c}\text { Network } \\
\text { Type }\end{array}$ & $\begin{array}{c}\text { Typical } \\
\text { Signs }\end{array}$ & $\begin{array}{c}\text { Different } \\
\text { Lighting } \\
\text { Condition }\end{array}$ & $\begin{array}{c}\text { Noisy } \\
\text { Signs }\end{array}$ \\
\hline $\begin{array}{c}\text { BP with } \\
\text { BAM }\end{array}$ & $98 \%$ & $95 \%$ & $96 \%$ \\
\hline BP & $89 \%$ & $78 \%$ & $82 \%$ \\
\hline
\end{tabular}

\subsection{Error Minimization}

In our training process, the program executed until the error comes to a minimum level. We can show how the error decreases per iteration by the following graph. Hence, at first we had implemented the task by using the BPNN algorithm and secondly, we had merged the BAM and BPNN algorithm to train and recognize the Road signs. We had observed the results and found that, using hybrid network (BAM and BPNN) rather than BPNN takes less iteration to train and less time to recognize signs.

We had taken 8 training input pattern and an error level (suppose 0.001) to stop the training process. The no. of iteration depends on the no. of hidden layer and also on what algorithm we used for the training process. Hence when we took the hidden layer $70 \%$ of the input layer, the iteration was 88043 but it decreased to 43067 when we used 50\% hidden layer.

When we used the hybrid network for the same percentage of hidden layer, the iteration decreased more than the previous rate. It decreased from 34075 to 14977.

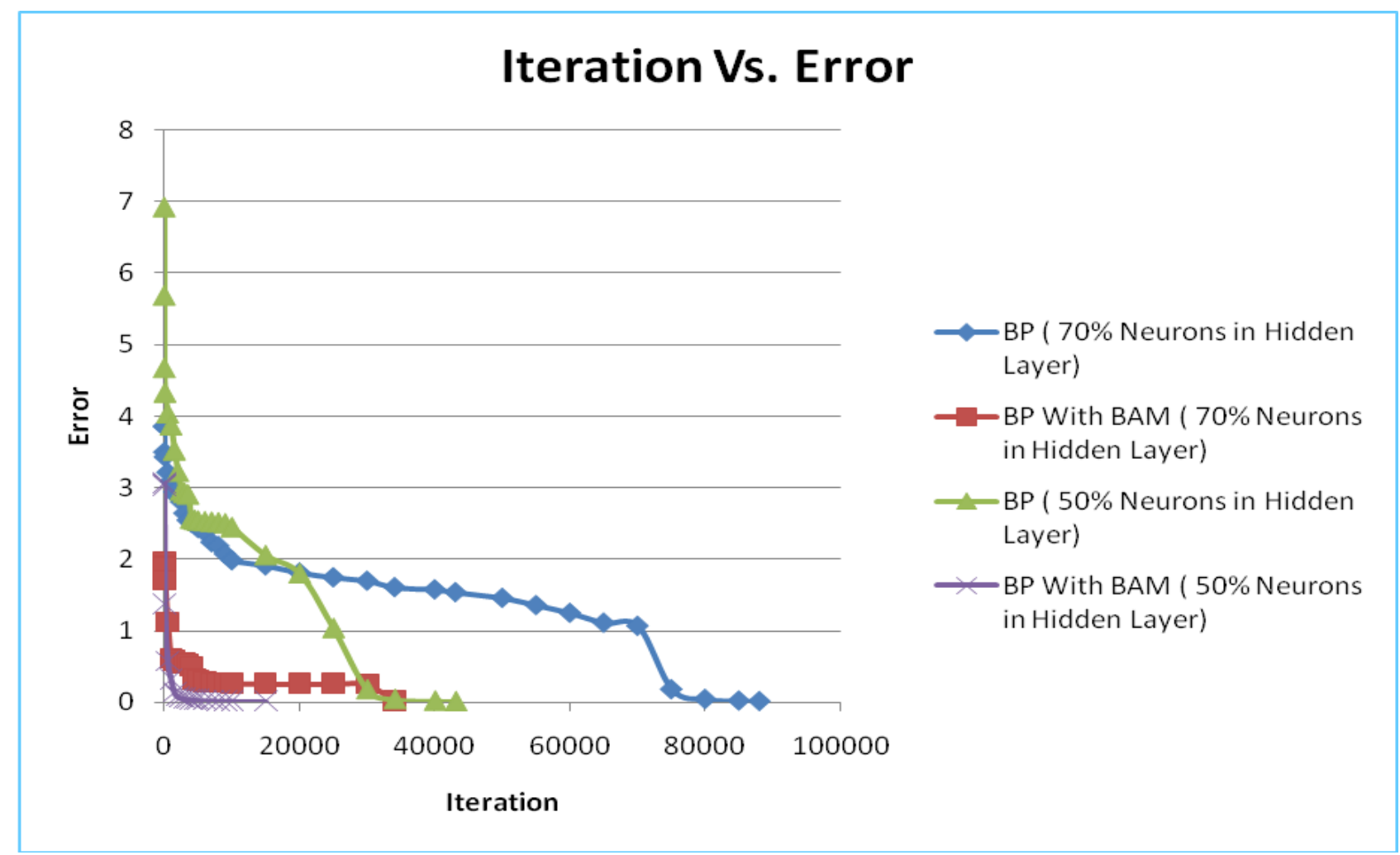

Comparing the performance of two networks as BPNN and Hybrid network we can take a decision that our considered hybrid network takes less iteration than BPNN in completion of the training process.

\section{CONCLUSION}

This research addresses the recognition of Road Signs using the BPNN and the hybrid (BAM+BPNN) network and make comparison in between this two network. We have presented a fast and robust system for sign recognition which is a combination of a local image sample representation and a hybrid network. The system we have presented is suitable one for a number of real-time applications. The system is capable of performing a classification in less than half a second for different classes of Road Signs. Hence we develop a generalized recognition system for Road Sign using the feature matrix $64 \times 64$ pixel. 


\section{REFERENCES}

[1] Haykin, S., 2001. "Neural Networks: A Comprehensive Foundation", 2nd Edition, Pearson Education Asia, pp 13-23.

[2] Mueller, R., Steck, M., 2003. "Road Sign Recognition”, Term Paper, Computer Perception with Artificial Intelligence, University of Applied Sciences, Biel, Switzerland.

[3] Piccioli, G., De Micheli, E., Parodi, P., Campani, M., 1996. "Robust Method for Road Sign Detection and Recognition", Image and Vision Computing 14, pp.208223.

[4] Novovicova, J., Paclik, P., Pudil, P., and Somol, P., 2000. "Road Sign Classification Using Laplace Kernel Classifier," Pattern Recognition Letters 21, pp. 11651173.

[5] Yuille, A. L., Snow, D., and Nitzberg,M., 1998. "Using Color to Detect, Localize and Identify Informational Signs", Proc. International Conference on Computer Vision ICCV98, Bombay, India, pp. 628-633.

[6] De la Escalera, A., Moreno, L., Salichs, M.A., and Amingol, J.M., 1997. "Road Traffic Sign Detection and Classification," IEEE Transactions Industrial Electronics, 44 , pp. 848-859.

[7] Lauziere, Y., Gingras, D., Ferrie, F., 2001. "A Modelbased Road Sign Identification System", Proc. IEEE Computer Conference on Computer Vision and Pattern Recognition, pp. 1163-1170.
[8] Shape-based road sign detection and recognition for embedded application using MATLAB Md Sallah, S.S.; Hussin, F.A.; Yusoff, M.Z.; Intelligent and Advanced Systems (ICIAS), 2010 Publication Year: 2010 , Page(s): $1-5$

[9] Fuzzy adaptive pre-processing models for road sign recognition. Chien-Chuan Lin; Ming-Shi Wang; TangChun Yang; Nature and Biologically Inspired Computing (NaBIC), 2010 Second World Congress on Publication Year: 2010 , Page(s): $642-647$

[10] Michael Shneier, 2005. "Road Sign Detection and Recognition", IEEE Computer Society International Conference on Computer Vision and Pattern Recognition.

[11] Sanjit Kumar Saha, Md. Shamsuzzaman, Prof. Dr. Md. Al-Amin Bhuiyan, 2010. "On Bangla Character Recognition", $13^{\text {th }}$ International Conference on Computer and Information Technology, pp. 436-439.

[12] Road sign detection and recognition system for real-time embedded applications. Sallah, S.S.M.; Hussin, F.A.; Yusoff, M.Z.; Electrical, Control and Computer Engineering (INECCE), 2011 Publication Year: 2011, Page(s): 213 - 218

[13] R. Ghica, S.Lu, and X.Yuan. Recogntion of traffic signs using a multilayer neural network. In Proc. Can Conf. On Electrical and Computer Engineering, 1994

[14] Gonzalez, "Digital Image Processing", $3^{\text {rd }}$ Edition, Pearson Education Asia, pp 336. 